\title{
Probabilistic Simulation of Shape Instability Based on the True Microstructure Model
}

\author{
G. C. Wu, Y. F. Li, ${ }^{1}$ and G. L. Wang \\ School of Mechanical Engineering, Harbin Institute of Technology, Harbin, China \\ 1 yuefengli@hit.edu.cn
}

Shape instability belongs to one of significant types of violation for disposable structural elements under high-stress levels. Due to lack of fundamental data on materials, it is quite problematic to consider the shape instability in the design of disposable structural elements. The crystal plastic finite element method is proposed to investigate the dispersion of shape instability life data. It allows these data to be obtained from traditional material parameters. The shape instability behavior is described with the constitutive crystal model of plastic damage accumulation. Then, to improve the accuracy of life prediction, the new method is developed to construct the simulation model of true microstructure. A modeling algorithm based on the image processing technology is provided to reduce the virtual stresses from the transient crystal plastic modeling method. Comparison of experimental and predicted results shows good agreement at high stresses close to the elastic limit of the material.

Keywords: shape instability failure, low-cycle fatigue, true microstructure, finite element analysis, disposable structural elements.

Introduction. Shape instability behavior has been commonly investigated as a special type of ratcheting, which mainly refers to the elastic-plastic dynamic transition process of materials. Shape instability is critical for disposable structural elements which are specially designed to undertake high overload cyclic stresses [1]. Therefore, the shape instability is identified via cyclic stress-strain curves as the state with $0.2 \%$ total plastic strain. In order to estimate the reliability of disposable structural elements, it is necessary to obtain the shape instability life scatter. Large scale tests are required. Moreover, as shape instability behavior only concerns the beginning stage of plastic deformation, the failure data have to be picked up from the cyclic stress-strain tests curves. The whole process is inefficient. Therefore, it is necessary to use a new efficient method to predict the shape instability failure.

Shape instability behavior is the result of multi plastic behavior of materials, such as low cycle fatigue damage, cyclic softening and ratcheting behavior [2-6]. Generally, the traditional crystal plastic model ignores the inhomogeneity of material properties at the microlevel [7-9]. It means that these models lack the ability to predict failure, depending on the main control factors of shape instability behavior. Therefore, in order to involve the material behavior mentioned above in the simulation model, a proper life prediction method based on the true microstructure model is developed for the shape instability failure prediction, which will provide a more accurate description on fatigue plastic deformation during the elastic-plastic transition. By choosing material parameters reasonably, probability crystal plastic finite element method is proved to be a wonderful way to investigate the dispersion of shape instability life. However, due to the differences between the true microstructure and simulated models, the simulation can hardly provide accurate distribution features, because the available method used in the simulation model is not accurate enough to simulate the complexity of microstructure. Therefore, in this study, a modeling algorithm based on the image processing technology is provided to solve this problem. 
1. Experimental Procedure and Modeling Method. Firstly, quenched and tempered $40 \mathrm{Cr}$ steel, which is a typical material used in the transmission elements, was chosen for this study. After quenching and tempering treatment, the hardness of hot-rolled $40 \mathrm{Cr}$ steel bars was about $\mathrm{HRC}=28-30$. The gauge diameter and length of specimens is machined into 12 and $25 \mathrm{~mm}$, respectively. After successively turning and polishing processes, the surface scratches of specimens could be removed.

Then, full tension-compression tests are conducted to identify material fatigue damage parameters in simulation models. Shape instability behavior tests are conducted to compare with the numerical model. Mean stress and stress amplitude are more frequently used in fatigue investigations, but not common parameters in the mechanical design. Therefore, adapt to mechanical design requirements, the maximum stress and stress ratio, calculated according to the maximum load and fatigue feature, are used as the control parameters in the tests.

Finally, to predict shape instability failure, a simulation model is established by using a proper material constitutive model, especially for the initial stage of fatigue process. The constitutive model will be present in Section 3. A geometric model similar to the true microstructure is also significant to reflect the microcyclic plastic strain and ratcheting strain. Therefore, this study proposed a method to build numerical models in the commercial finite element analysis software ABAQUS. As shown in Fig. 1, firstly, the true microstructure of steel $40 \mathrm{Cr}$ is obtained by using electron backscatter diffraction measurements. Then, the grain boundary could be preliminary identified based on the image processing technology. By doing this, the chromatic image is transformed to a new gray-scale image. Actually, the crystal orientation information is abandoned in this process, because it is not necessary to accurately copy the crystal orientation in the numerical model. At this time, the grain boundaries are not clear enough to exact single grains. Therefore, a denoising process is essential to delete unreasonable lines. A unique requirement in this stage is that the lines forming grains should be closed; otherwise, the open lines will bring mistakes in the grains geometry model restructure process. Finally, the independent grains with clear boundaries are exacted. The topography information could be output as the required format of input file by ABAQUS. The geometry model is similar with the true microstructure of materials.

After determining the model scale, the performances of grains in the model, including the elastic module and the yield strength of the grains, are directly obtained via nanoindentation tests or indirectly determined from stress-strain curve referring to the method provided by Liu and Chen [10]. Figure 2 shows the fifty random position points used to conduct the tests on the specimen. According to these material data, Gaussian distribution parameters could be fitted. For the steel $40 \mathrm{Cr}$ specimens in this study, the elastic limit had an expected value $790 \mathrm{MPa}$ and standard deviation $92 \mathrm{MPa}$, respectively.

2. Simulation. For the purpose of predicting the shape instability failure, it is essential to investigate shape instability behavior and estimate shape instability life by using a proper model based on damage-coupled cyclic plasticity theory. According to the determination rule of shape instability failure, $0.2 \%$ total plastic strain occurs in the initial stage of the fatigue process, which contains cyclic plastic strain and ratcheting strain. Therefore, the cyclic plastic behavior of metallic materials is described based on the frame of FrederickArmstrong model [11]. The evolution law supposed by Ohno and Wang is used to avoid the overestimation of ratcheting strain in the initial stage of cyclic plastic behavior $[12,13]$. The elastic-plastic fatigue damage model has been provided by Lemaitre and Chaboche [14]. By utilizing the UMAT function offered in the software ABAQUS, damaged-coupled cyclic crystal plastic constitutive numerical equations was programmed, which was given in our previous work $[15,16]$. The main control equations are simply presented as followed:

The total strain $\varepsilon_{i j}$ is as follows:

$$
\varepsilon_{i j}=\varepsilon_{i j}^{e}+\varepsilon_{i j}^{p} .
$$




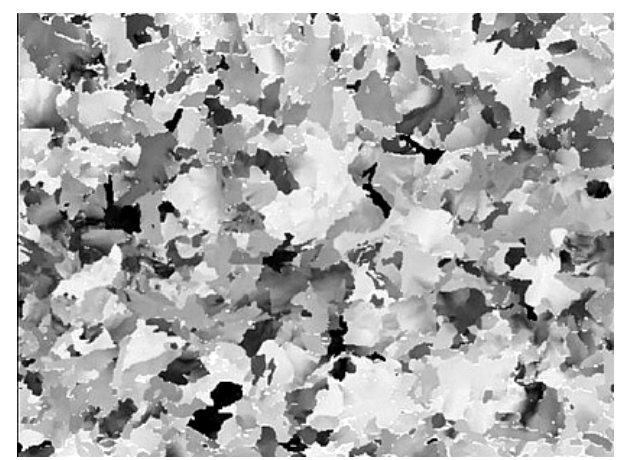

$\mathrm{a}$

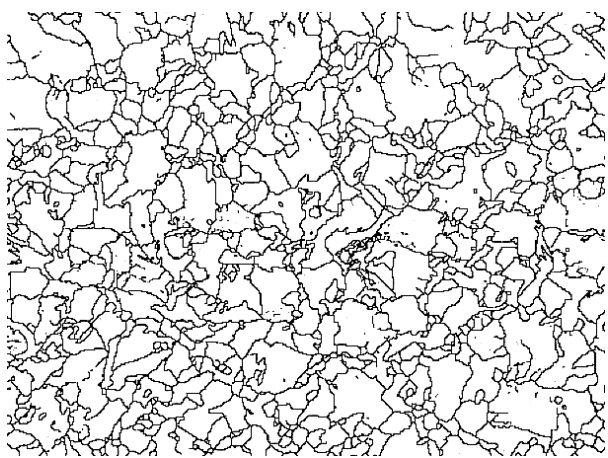

$\mathrm{c}$

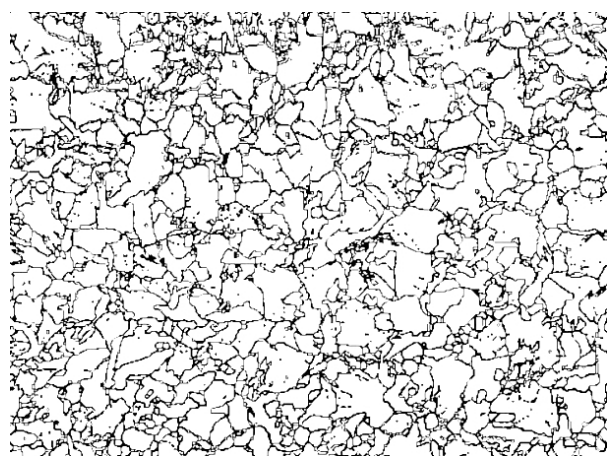

b

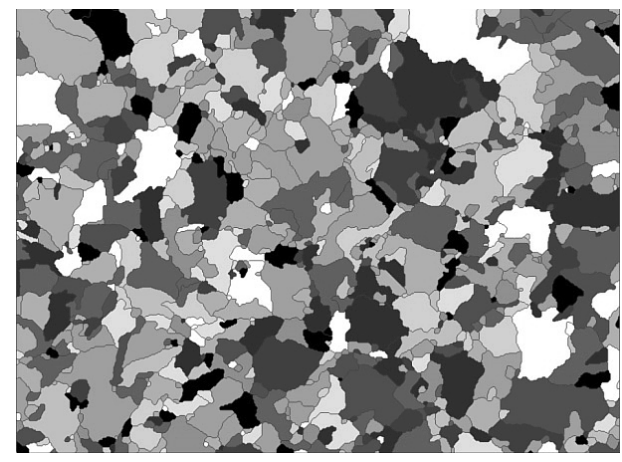

d

Fig. 1. The procedure to build a geometric model according to EBSD test figure: (a) EBSD figure; (b) grain boundary initial identification; (c) grain boundary processing; (d) grain exaction and topological information output.

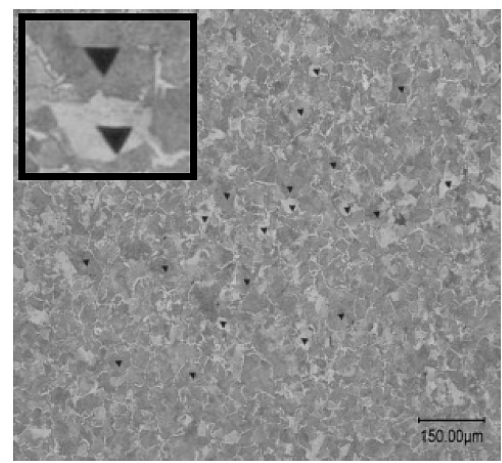

Fig. 2. Nano-indentation test conducted at 50 random points to obtain grain mechanics performances.

The elastic strain, $\varepsilon_{i j}^{e}$, is expressed as

$$
\varepsilon_{i j}^{e}=\frac{1+v}{E}\left(\frac{\sigma_{i j}}{1-D}\right)-\frac{v}{E}\left(\frac{\sigma_{k k} \delta_{i j}}{1-D}\right),
$$

where $v$ is Poisson's ratio, $E$ is elastic modulus, $\sigma_{i j}$ is Cauchy stress tensor, $D$ is damage parameter, $\sigma_{k k}$ is the main diagonal component of $\sigma_{i j}$, and $\delta_{i j}$ are Kronecker delta. 
The evolution law of plastic strain, $\bar{\varepsilon}_{i j}^{p}$, is expressed as

$$
\bar{\varepsilon}_{i j}^{p}=\lambda \frac{\partial F}{\partial \sigma_{i j}},
$$

where $\lambda$ is a non-negative scalar.

The Mises yield function with damage $F$ is given as follows:

$$
F=\left(\frac{3}{2}\left(\frac{\sigma_{d e v}}{1-D}-a\right):\left(\frac{\sigma_{d e v}}{1-D}-a\right)\right)^{1 / 2}-Q
$$

where $\sigma_{d e v}$ is the deviatoric stress tensor and $a$ is the backstress tensor in deviatoric space.

The yield surface $Q$ is expressed as

$$
\bar{Q}=\bar{p}\left(Q_{\infty}-Q\right) b_{1},
$$

where $Q_{\infty}$ and $b_{1}$ are material constants, $\bar{p}$ is given as

$$
\bar{p}=\left(\frac{2}{3}\left(\bar{\varepsilon}_{i j}^{p}: \bar{\varepsilon}_{i j}^{p}\right)\right)^{1 / 2}=\frac{\lambda}{1-D} .
$$

The back stress tensor $a$ in the Eq. (4) is expressed as

$$
a=\sum_{i=1}^{m} a^{(i)} \quad(i=1,2, \ldots, n)
$$

where $m$ is the backstress component number, $a^{(i)}$ is given as

$$
\begin{gathered}
\bar{a}^{(i)}=(1-D) c^{(i)}\left[\frac{2}{3} r^{(i)} \bar{\varepsilon}^{p}-\left(\frac{\left\|\chi^{(i)}\right\|}{r^{(i)}}\right)^{n}\left\langle\bar{\varepsilon}^{p}: \frac{a^{(i)}}{\left\|a^{(i)}\right\|}\right) a^{(i)}\right], \\
\left\|a^{(i)}\right\|=\sqrt{a^{(i)}: a^{(i)}}
\end{gathered}
$$

where $c^{(i)}, r^{(i)}$, and $n$ are material constants.

The damage parameter, $D$, is expressed as follows:

$$
\frac{\partial D}{\partial N}=\left[1-(1-D)^{1+\beta}\right]^{\alpha}\left(\frac{A_{I I}}{M_{0}\left(1-3 b_{2} \sigma_{\text {mean }}\right)(1-D)}\right)^{\beta},
$$

where $\alpha, \beta, M_{0}$, and $b_{2}$ are material constants, $\sigma_{\text {mean }}$ is the mean value belonging to hydrostatic stress, which is zero, and $A_{I I}$ is the parameter determined by the Sines yield criterion.

On the basis of the shape instability life character, the heterogeneity of materials needs to be considered in the prediction of shape instability failure. The heterogeneity of a material mainly includes two aspects, the microstructure features and the grain mechanics 
properties. Therefore, a two-dimensional probabilistic crystal cyclic plastic finite element model is built to predict shape instability failure.

Figure 3 shows the process to copy the true microstructural and grain performances in the simulation model to predict the shape instability failure prediction in ABAQUS. As shown in Fig. 3a, representative elementary volume (RVE) with different stochastic microstructures are established in ABAQUS. Figure $3 \mathrm{~b}$ shows the method to assign random material performances in the RVE model. Based on the obtained Gaussian distribution in Section 2, mechanics properties are randomly produced, applying to assign to the grains in RVE. After endowing the elements with the fatigue damage-coupled cyclic plastic material constitutive model, the simulation model could present mechanics properties with the true specimens on the microlevel. As the morphology and the grain properties of RVE are both randomly generated, the failure could be simulated by using different random numbers. The simulation results will reflect the material mechanical property dispersion on a macrolevel, which is accordance with the practical situation.

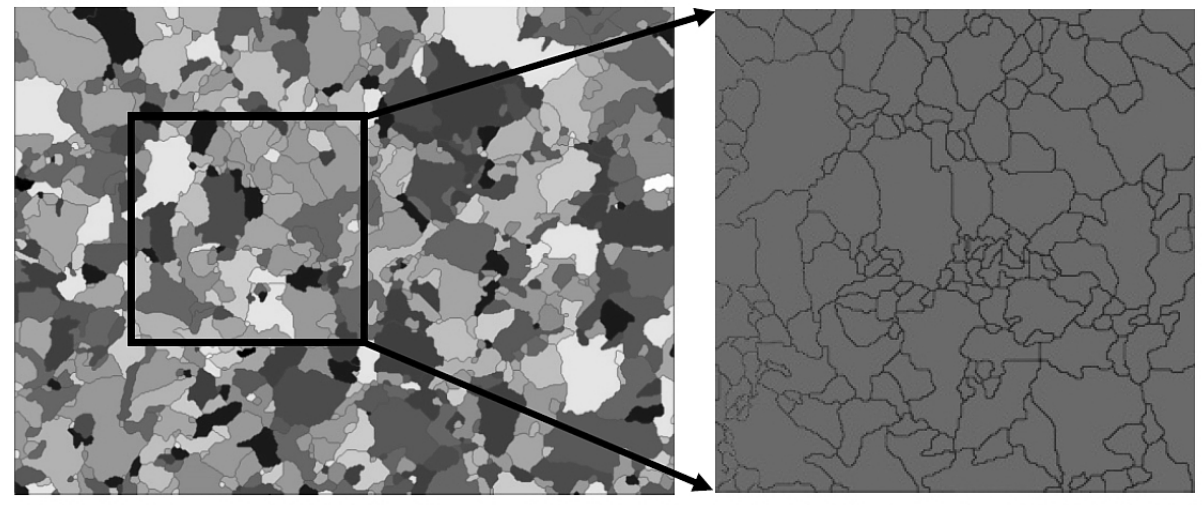

a

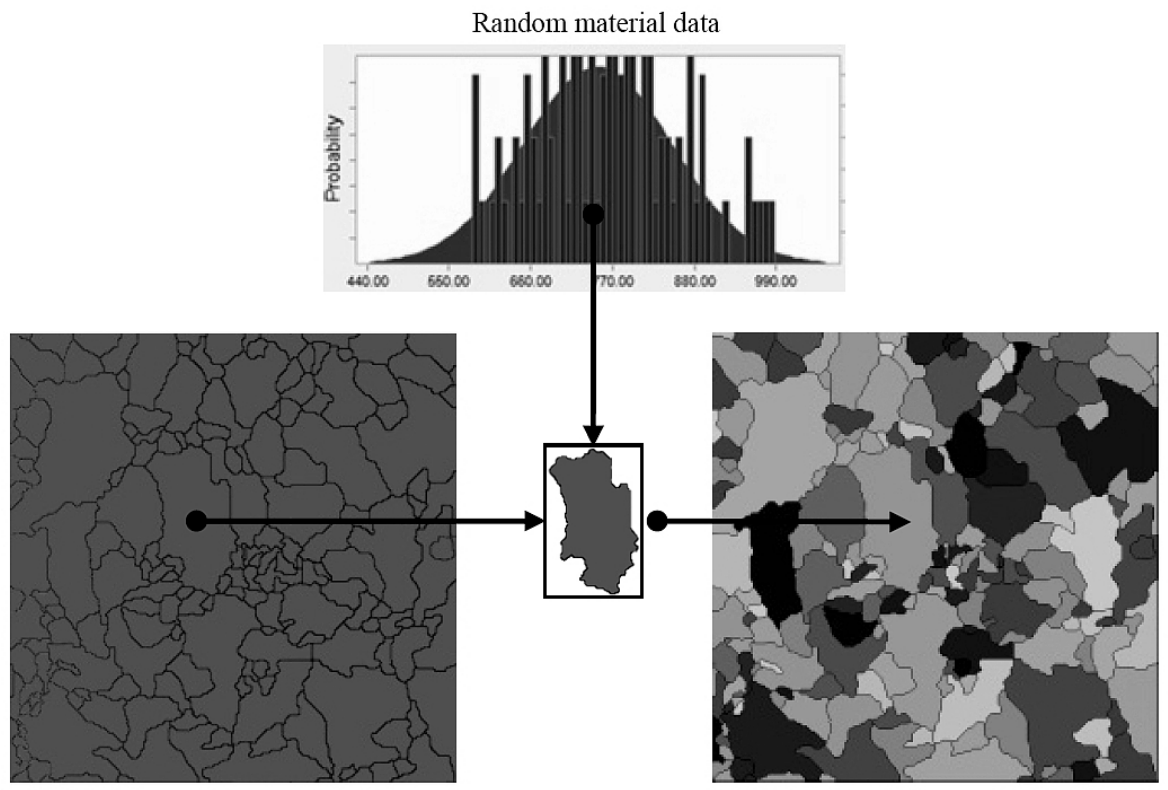

b

Fig. 3. Schematic showing the method used to simulate the probabilistic failures of shape instability behavior: (a) the simulation model selected from any part of the whole model; (b) the stochastic performances of grains based Monte-Carlo method. 
3. Results and Discussion. Generally, the influence level of artificial stresses in the simulation model is difficult to evaluate, because they are coupled with many other factors. Therefore, the model based on the Voronoi tessellation is usually established based on the uniform distribution to avoid artificial stresses. However, it will ignore the complexity of microstructure. The new modeling method could obviously decrease the differences between simulation models and true microstructure. By doing this, the problem to evaluate artificial stresses is technically bypassed and then solved.

Figure 4 compares the simulated life scatter with the experiment results. The stress-strain curves with $0.2 \%$ total plastic strain are picked out from these hysteresis curves obtained from the experiments or the simulations. It can be seen that the required number of cycles needed to reach $0.2 \%$ plastic strain is different between the experiments and the simulations. The differences are a result of the stochastic simulation procedure. To obtain the life scatter band, a group of material properties is randomly generated based on a certain distribution. These material properties are also randomly assigned in the simulation model elements. The overall performance of the simulation models vary with each simulation. These variations yield the life scatter, but they also lead to differences between the simulation and the experimental results in one certain simulation calculation. If the stochastic procedure is removed from the simulation model, then results identical to the experimental data can be obtained by using suitable material parameters; the constitutive models used in this paper have been validated in many studies [17-19]. As the figure shows, the prediction model is more accurate at high-stress levels than a low-stress levels, especially at $0.95 \sigma_{s}$. Errors occur between the simulation and the experiment when the maximum stress was at $0.7 \sigma_{s}$. The errors come from the damage parameter, $D$, used in the constitutive model. Steel $40 \mathrm{Cr}$ presents high cycle fatigue features at $0.7 \sigma_{s}$ maximum stress. However, $D$ is determined based on the assumption of low-cycle fatigue statuses, which means that larger $D$ values are used in the constitutive model. Therefore, the simulation shape instability life is shorter than that determine experimentally. Although the errors are at low-stress levels, the simulation method provides accurate prediction results for shape instability life at high-stress levels, which is more significant for applications in disposable structural elements design. Therefore, the deviation could be ignored when it is at low-stress levels.

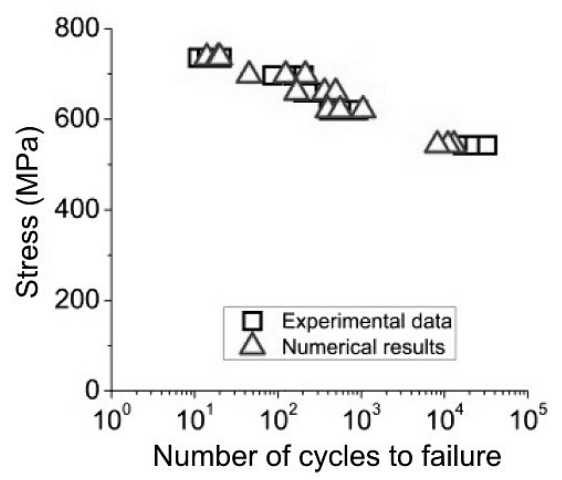

Fig. 4. Comparison between the experiment and the simulated results of $40 \mathrm{Cr}$ steel life predictions.

Conclusions. Disposable structural elements are rarely mentioned but widely applied in aerospace vehicles. Based on the high payload requirements, disposable structural elements usually undertake higher stress levels than general structural elements. Under these circumstances, shape instability failure is significantly increased. Therefore, this study provides a numerical simulation method based on the true microstructure model for the shape instability failure. A modeling method based on the image processing technology 
is developed to simulate the true microstructure. The comparison between the experiments and predictions shows good agreement in the concerned process. By doing this, the numbers of shape instability life tests could be significantly decreased.

Acknowledgments. National Natural Science Foundation of China (51405101), the research and innovation fund of Harbin Institute of Technology (Grant Number HIT.NSRIF.2015 053), the China Postdoctoral Science Foundation (Grant Numbers 2014M561340 and 2016T90277) and Heilongjiang Postdoctoral Fund (Grant Number LBH-Z14100).

1. Y. F. Li, X. D. Pan, and G. L. Wang, "Low cycle fatigue and ratcheting properties of steel 40Cr under stress controlled tests," Int. J. Fatigue, 55, 74-80 (2013).

2. S. J Park, K. S. Kim, and H. S. Kim, "Ratcheting behavior and mean stress considerations in uniaxial low-cycle fatigue of Inconel 718 at $649^{\circ} \mathrm{C}$," Fatigue Fract. Eng. Mater. Struct., 30, No. 11, 1076-1083 (2007).

3. C. B. Lim, K. S. Kim, and J. B. Seong, "Ratcheting and fatigue behavior of a copper alloy under uniaxial cyclic loading with mean stress," Int. J. Fatigue, 31, No. 3, 501-507 (2009).

4. G. Z. Kang, Y. J. Liu, J. Ding, and Q. Gao, "Uniaxial ratcheting and fatigue failure of tempered 42CrMo steel: Damage evolution and damage-coupled viscoplastic constitutive model," Int. J. Plasticity, 25, No. 5, 838-860 (2009).

5. H. Lim, J. D. Carroll, C. C. Battaile, et al., "Grain-scale experimental validation of crystal plasticity finite element simulations of tantalum oligocrystals," Int. J. Plasticity, 60, 1-18 (2014).

6. D. Yu, K. An, Y. Chen, and X. Chen, "Revealing the cyclic hardening mechanism of an austenitic stainless steel by real-time in situ neutron diffraction," Scripta Mater., 89, 45-48 (2014).

7. S. Keshavarz and S. Ghosh, "Multi-scale crystal plasticity finite element model approach to modeling nickel-based superalloys," Acta Mater., 61, No. 17, 6549-6591 (2013).

8. M. Naderi, S. H. Hoseini, and M. M. Khonsari, "Probabilistic simulation of fatigue damage and life scatter of metallic components," Int. J. Plasticity, 43, 101-115 (2013).

9. S. Ghosh and M. Anahid, "Homogenized constitutive and fatigue nucleation models from crystal plasticity FE simulations of Ti alloys, Part 1: Macroscopic anisotropic yield function," Int. J. Plasticity, 47, 182-201 (2013).

10. Y. Liu and D. Chen, "Measurement of material mechanical properties using nanoindentation and finite element simulation," J. Wuhan Univ. Technol., 27, 690-693 (2003).

11. C. O. Frederick and P. J. Armstrong, A Mathematical representation of the Multiaxial Bauschinger Effect, Report RD/B/N 731, Central Electricity Generating Board (1966).

12. N. Ohno and J.-D. Wang, "Kinematic hardening rules with critical state of dynamic recovery, part I: Formulation and basic features for ratchetting behavior," Int. J. Plasticity, 9, No. 3, 375-390 (1993).

13. N. Ohno and J.-D. Wang, "Kinematic hardening rules with critical state of dynamic recovery, part II: application to experiments of ratchetting behavior," Int. J. Plasticity, 9, No. 3, 391-403 (1993).

14. J. Lemaitre and J.-L. Chaboche, Mechanics of Solid Materials, Cambridge University Press, Cambridge, UK (1990). 
15. Y. F. Li, X. D. Pan, G. C. Wu, and G. L. Wang, "Shape-instability life scatter prediction of $40 \mathrm{Cr}$ steel: Damage-coupled crystal plastic probabilistic finite element method," Int. J. Plasticity, 79, 1-18 (2016).

16. G. C. Wu, Y. F. Li, X. D. Pan, and G. L. Wang, "Numerical simulation of fatigue damage and shape instability behavior of steel $40 \mathrm{Cr}$ by the damage-coupled crystal plastic model," Strength Mater., 49, No. 1, 118-124 (2017).

17. N. Ohno, "Constitutive modeling of cyclic plasticity with emphasis on ratcheting," Int. J. Mech. Sci., 40, Nos. 2-3, 251-261 (1998).

18. N. Ohno, M. Abdel-Karim, M. Kobayashi, and T. Igari, "Ratchetting characteristics of 316FR steel at high temperature, part I: Strain-controlled ratchetting experiments and simulations," Int. J. Plasticity, 14, Nos. 4-5, 355-372 (1998).

19. N. Ohno and M. Abdel-Karim, "Uniaxial ratchetting of 316FR steel at room temperature - II: Constitutive modeling and simulation," J. Eng. Mater. Technol., 122, No. 1, 35-41 (2000).

Received 15. 09. 2017 